\title{
Factorization of standard model cross sections at ultrahigh energy
}

\author{
Yang-Ting Chien ${ }^{1, *}$ and Hsiang-nan $\mathrm{Li}^{2, \dagger}$ \\ ${ }^{1}$ Center for Theoretical Physics, Massachusetts Institute of Technology, \\ Cambridge, Massachusetts 02139, USA \\ ${ }^{2}$ Institute of Physics, Academia Sinica, Taipei, Taiwan 115, Republic of China
}

(Received 18 January 2018; published 26 March 2018)

\begin{abstract}
The factorization theorem for organizing multiple electroweak boson emissions at future colliders with energy far above the electroweak scale is formulated. Taking the inclusive muon-pair production in electron-positron collisions as an example, we argue that the summation over isospins is demanded for constructing the universal distributions of leptons and gauge bosons in an electron. These parton distributions are shown to have the same infrared structure in the phases of broken and unbroken electroweak symmetry, an observation consistent with the Goldstone equivalence theorem. The electroweak factorization of processes involving protons is sketched, with an emphasis on the subtlety of the scalar distributions. This formalism, in which electroweak shower effects are handled from the viewpoint of factorization theorem for the first time, is an adequate framework for collider physics at ultra high energy.
\end{abstract}

DOI: 10.1103/PhysRevD.97.053006

The discovery of the Higgs boson is one of the recent greatest achievements in particle physics. The precise determination of Higgs boson properties, the deeper understanding of the electroweak (EW) symmetry breaking mechanism, and the continuous search for physics beyond the standard model (SM) then motivate the construction of next-generation colliders, such as the Super Proton-Proton Collider [1,2] and the Future Circular Collider [3]. The SM dynamics at energy $E$ far above the EW scale, i.e., the vacuum expectation value (VEV) $v$ of the Higgs field, produces new phenomena [4] and impacts heavy dark matter searches [5-8]. Multiple emissions of EW gauge bosons enhanced by the large radiative logarithms $\ln (v / E)$ imply the invalidity of fixed-order calculations $[9,10]$. It demands the treatment of EW gauge bosons as partons in a factorization theorem, which inevitably alters the conventional framework for SM studies. In this paper we will construct a formalism adequate for collider physics at ultra high energy, in which the EW shower effects are organized from the viewpoint of factorization.

The EW sector shares features similar to those of QCD but also exhibits fundamental differences. The dynamically broken chiral symmetry in QCD gives rise to hadron

\footnotetext{
*ytchien@mit.edu

†hnli@phys.sinica.edu.tw
}

Published by the American Physical Society under the terms of the Creative Commons Attribution 4.0 International license. Further distribution of this work must maintain attribution to the author(s) and the published article's title, journal citation, and DOI. Funded by SCOAP ${ }^{3}$. masses, while the spontaneously broken EW symmetry induces particle masses. The VEV $v$ plays a role similar to the QCD scale $\Lambda_{\mathrm{QCD}}$, so that $v / E$ defines a power expansion parameter in the analysis of infrared EW radiation. The isospin and hypercharge in the EW sector correspond to the color in QCD. Due to the confinement, only color-singlet bound states can be prepared and detected in experiments. We need to sum over colors when connecting parton- and hadron-level observables in the QCD factorization. However, we can distinguish particle flavors and thus encounter the violation of the BlockNordsieck theorem $[11,12]$. The aforementioned infrared EW logarithms $\ln (v / E)$ then renders the parton- and particle-level connection nontrivial.

We propose that the logarithms $\ln (v / E)$ should be handled in a way similar to the QCD factorization for the infrared logarithms $\ln \left(\Lambda_{\mathrm{QCD}} / E\right)$ : the collinear logarithms are factorized into parton distribution functions (PDFs), and the soft logarithms cancel to ensure the universality of the PDFs. Nevertheless, there exists additional subtlety in the EW factorization: in the high energy limit the mass term in the Higgs potential becomes irrelevant with the approximate restoration of the EW symmetry. It is also possible that a new ultraviolet theory with the exact EW symmetry exists, which generates the SM Higgs potential radiatively at low energy [13]. In either case, we can consider an EW symmetry breaking scale $\mu_{s}$, above which the EW symmetry is restored, and EW corrections contain infrared divergences. Such restoration does not exist for the chiral symmetry in zero-temperature QCD. With the additional scale $\mu_{s}$, as well as scalar 
emissions through the Yukawa couplings in a huge hierarchy, the power counting for the EW factorization is expected to be more complicated than for the QCD one.

We analyze the EW infrared structure of the inclusive production of muon pairs in electron-positron collisions $e^{-} e^{+} \rightarrow \mu^{-} \mu^{+}+X$, and examine how EW emissions modify the PDF definitions of an electron. It is shown that a well-defined PDF at a scale $E \gg v$ requires the summation over the $S U(2)_{L} \times U(1)_{Y}$ charges, i.e., isospins of partons. Adding EW shower directly into the PDFs [14-16] originally designed to absorb QCD nonperturbative dynamics works only for $E$ not far above $v$, where the EW radiation is not yet significant enough to violate the universality. Furthermore, we argue that the PDFs have the same infrared structure in the EW symmetry unbroken and broken phases, so it suffices to implement a perturbative matching for the PDFs in the two phases at the scale $\mu_{s}$. The EW factorization theorem then provides a unified handle of the dynamics above and below $\mu_{s}$ up to power corrections in $v / E$. It also allows us to resum the large logarithms $\ln (v / E)$, a subject to be explored in the future.

For simplicity, we focus on the first lepton generation and turn off the EW emissions from the muons. The construction of fragmentation functions [6,17] follows the same reasoning. The next-to-leading order (NLO) corrections $[18,19]$ to the leading-order (LO) process in the broken phase are displayed in Fig. 1. The universality of the PDFs holds only if the soft logarithms from gauge boson exchanges, which couple the initial electron and positron beams, cancel between the virtual and real corrections. The soft cancellation is straightforward in the case, where a photon or a $Z$ boson attaches to the incoming partons $e^{-}$and $e^{+}$. In the virtual correction with the $W^{-}$boson exchange, the parton $e^{-}$on the left side of the final state cut emits $W^{-}$, becoming a neutrino $\nu_{e}$, and the parton $e^{+}$absorbs $W^{-}$, becoming an anti-neutrino $\bar{\nu}_{e}$. For the real correction, the other incoming parton must be $\bar{\nu}_{e}$ produced through EW shower of $e^{+}$in order to annihilate $\nu_{e}$ into a $Z$ boson. To cancel the soft logarithms, the above two NLO diagrams with different partons $e^{+}$and $\bar{\nu}_{e}$ have to be added into the isospin $I=0$ combination, following the
EW version of the Kinoshita-Lee-Nauenberg theorem $[20,21]$. It indicates that we can define only the lepton distribution of the positron, $\phi_{\ell / e}$ : a positron and an antineutrino can frequently convert to each other by emitting $W$ bosons and form a lepton beam before entering the hard process, to which both components of the lepton doublet contribute.

As in the QCD factorization, collinear gauge bosons are factorized by eikonalizing the parton lines the gauge bosons attach to, giving the Wilson links which appear in the definition of the lepton PDF as a matrix element of a nonlocal operator. Wilson links are crucial for the gauge invariance of a PDF. Certainly, the diagrams with collinear gauge bosons attaching to hard particle lines should be included to complete the factorization. Scalar emissions from the initial lepton in two-particle irreducible diagrams (like those in Fig. 1) are free of leading-power collinear divergences, since the product of two adjacent on-shell fermion propagators suppresses the collinear region. It means that collinear scalars do not contribute to Wilson links, namely, are irrelevant to the gauge invariance. Scalar emissions in two-particle reducible diagrams, such as the self-energy correction of the lepton, can generate leadingpower infrared logarithms, which are, however, suppressed by the Yukawa coupling in the present study.

For the construction of gauge boson PDFs, the parton $e^{+}$is replaced by a transversely polarized $W^{+}$boson from EW emissions. The contribution from a longitudinally polarized $W^{+}$boson is power-suppressed. The corresponding LO diagram is shown in the left panel of Fig. 2, where the flavor is labeled explicitly for each line. At NLO, the exchange of a photon or a $Z$ boson has straightforward soft cancelation. The exchanges of a virtual $W^{-}$boson and a real $W^{-}$boson are exhibited in the middle and right panels of Fig. 2, respectively. The soft cancelation demands the summation of the two NLO diagrams and the construction of the charged boson distribution, $\phi_{W / e}$. Scalar emissions by a $W$ boson are power-suppressed by $v / E$ compared to gauge boson emissions, so Wilson links associated with the $W$ boson PDF do not collect collinear scalars either.
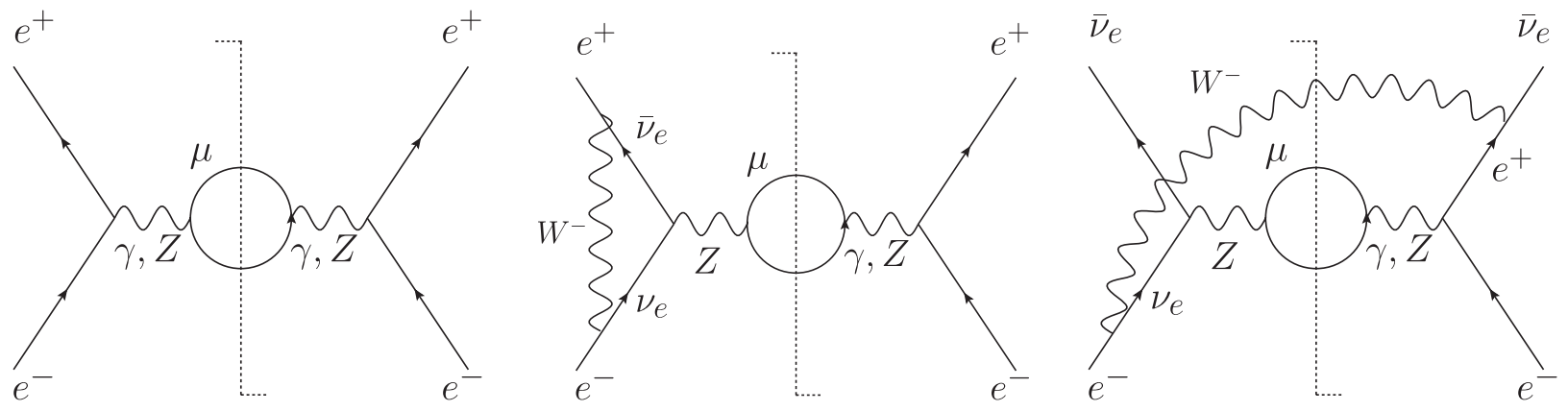

FIG. 1. The LO diagram of the process $e^{+} e^{-} \rightarrow \mu^{+} \mu^{-}+X$ (left panel) and the NLO corrections with the $W^{-}$boson exchange (middle and right panels) at the parton level. The dashed lines represent the final state cuts. 

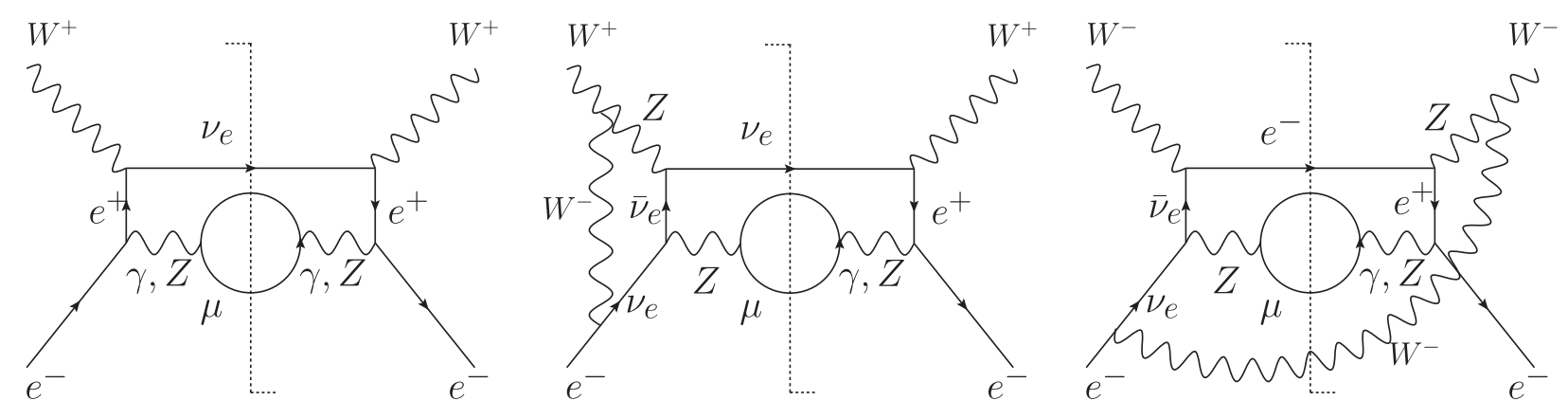

FIG. 2. The LO diagram involving the parton $W^{ \pm}$of $e^{+}$(left panel), and its NLO corrections with the $W^{-}$boson exchange (middle and right panels).

The construction of the photon and $Z$ boson distributions is more involved. The incoming parton can be either a photon or a $Z$ boson on each side of the final state cut, so we need to consider the $\gamma-\gamma, \gamma-Z, Z-\gamma$ and $Z-Z$ combinations. The virtual $W^{-}$corrections in Fig. 3 contribute to all the combinations, while the real $W^{-}$corrections contribute only to the $Z-\gamma$ and $Z-Z$ combinations. To have the soft cancelation, we must include the partner parton $\nu_{e}$ of $e^{-}$. The soft logarithms in the virtual $W^{-}$correction to the $e^{-}$-initiated process then cancel those in the real $W^{+}$correction to the $\nu_{e}$-initiated process. Similarly, the soft logarithms in the real $W^{-}$correction to the $Z-\gamma$ and $Z-Z$ combinations in the $e^{-}$-initiated process cancel those in the virtual $W^{+}$correction to the $\gamma-Z$ and $Z-Z$ combinations in the $\nu_{e}$-initiated process. For the $Z$ boson exchanges at NLO, the virtual $Z$ contributes to the $Z-\gamma$ and $Z-Z$ combinations, while the real $Z$ contributes to the $\gamma-Z$ and $Z-Z$ combinations, among which the soft logarithms also cancel. The above reasoning leads to the definitions of the photon, the $Z$ boson and the mixed PDFs, $\phi_{\gamma / e}, \phi_{Z / e}$ and $\phi_{\gamma Z / e}$, respectively.

The lepton distribution $\phi_{\ell / e}(x, \mu)$ of the electron is written as

$$
\begin{aligned}
\phi_{\ell / e}(x, \mu)= & \frac{1}{4} \sum_{s} \int \frac{d y^{-}}{2 \pi} \exp \left(-i x p^{+} y^{-}\right) \\
& \times\langle e(p, s)| \sum_{\ell=e, \nu_{e}} \bar{\ell}\left(y^{-}\right) W^{\dagger}\left(y^{-}\right) \\
& \times \frac{1}{2} \gamma^{+} W(0) \ell(0)|e(p, s)\rangle,
\end{aligned}
$$

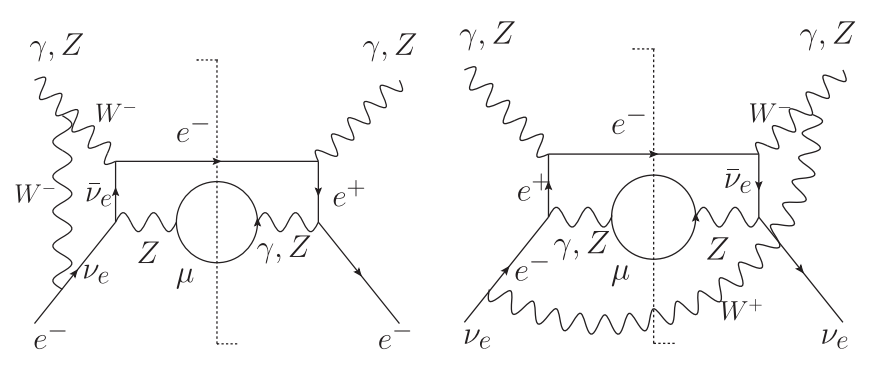

where $x$ is the momentum fraction, $\mu$ is the factorization scale, and the Wilson link $W(y)$ is defined as

$$
\begin{aligned}
W(y)= & P\left(\exp \left[i g \int_{0}^{\infty} d z n \cdot W_{i}(y+z n) \sigma_{i}\right]\right. \\
& \left.\times \exp \left[i g^{\prime} \int_{0}^{\infty} d z n \cdot B(y+z n) I\right]\right),
\end{aligned}
$$

with the Pauli matrices $\sigma_{i}$, the identity matrix $I$, the $S U(2)_{L} \times U(1)_{Y}$ gauge fields $W_{i}(i=1,2,3)$ and $B$, and the gauge couplings $g$ and $g^{\prime}$ of $S U(2)_{L}$ and $U(1)_{Y}$, respectively. The gauge eigenstates and the mass eigenstates can be transformed into each other, $W_{1,2} \leftrightarrow W^{ \pm}$and $\left(W_{3}, B\right) \leftrightarrow(Z, \gamma)$, but the expression will be more complicated if written in terms of the latter due to the distinctive couplings. It has been found that the difference between the up- and down-type fermion distributions (the isospin $I=1$ piece, which does not respect the universality) is driven to diminishing by a double-logarithmic EW evolution $[15,16]$. This result supports that only the $I=0$ piece in Eq. (1) is needed for the EW factorization, whose associated hard function sums the contributions from both isospins. The $W$ boson distribution is given by

$$
\begin{aligned}
\phi_{W / e}(x, \mu)= & \frac{1}{2} \sum_{s} \int \frac{d y^{-}}{2 \pi x p^{+}} \exp \left(-i x p^{+} y^{-}\right) \\
& \times\langle e(p, s)| \sum_{i=1,2} W_{i \nu}^{+}\left(y^{-}\right) W^{\dagger}\left(y^{-}\right) \\
& \times W(0) W_{i}^{\nu+}(0)|e(p, s)\rangle .
\end{aligned}
$$

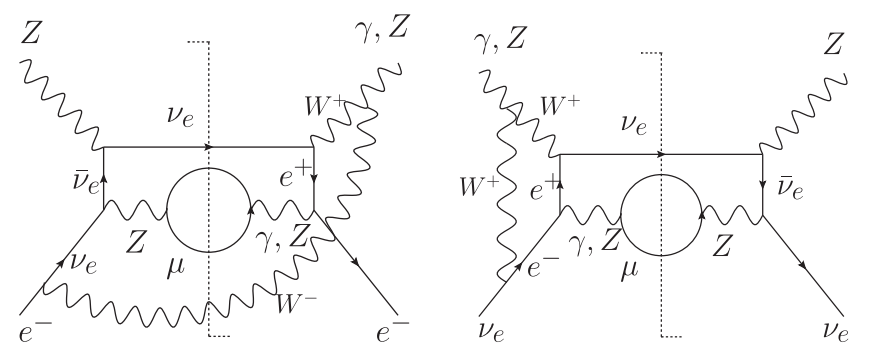

FIG. 3. The NLO diagrams involving the parton $\gamma$ or $Z$ of $e^{+}$, with the $W$ boson exchange. 
The definitions for the photon, the $Z$ boson and the mixed distributions are similar, with the field tensor $W_{i}^{\mu \nu}$ replaced by the corresponding ones.

When the factorization scale runs above the symmetry breaking scale $\mu_{s}$, all particles become massless and some scalar degrees of freedom emerge. It is then a concern how the infrared structure of the process and the PDFs are modified. Below we argue that the PDFs in the symmetry unbroken phase contain the same collinear logarithms as in the symmetry broken phase. For a massive gauge boson of momentum $k$ in the broken phase, we adopt the propagator

$$
\frac{-i}{k^{2}-m_{W}^{2}}\left[g^{\mu \nu}-\left(1-\frac{1}{\lambda}\right) \frac{k^{\mu} k^{\nu}}{k^{2}-m_{W}^{2} / \lambda}\right]
$$

in the covariant gauge with the gauge parameter $\lambda$. For a massless gauge boson in the unbroken phase, we use

$$
\frac{-i}{k^{2}-m_{W}^{2}}\left[g^{\mu \nu}-\left(1-\frac{1}{\lambda}\right) \frac{k^{\mu} k^{\nu}}{k^{2}}\right],
$$

where the physical $W$ boson mass $m_{W}$ serves as an infrared regulator. The PDFs, being gauge invariant, can be computed in an arbitrary gauge, and we choose the Feynman gauge $\lambda \rightarrow 1$. The metric tensors for real gauge boson emissions are given by

$$
g^{\mu \nu}-\frac{k^{\mu} k^{\nu}}{m_{W}^{2}}, \quad g^{\mu \nu}-\frac{k^{\mu} \bar{k}^{\nu}+k^{\nu} \bar{k}^{\mu}}{k \cdot \bar{k}},
$$

in the broken and unbroken phases, respectively, with $k=\left(k^{0}, \vec{k}\right)$ and $\bar{k}=\left(k^{0},-\vec{k}\right)$. There exists cancelation with the corresponding virtual diagrams in the soft region. The momentum $k$ picks up the minus lightcone component of the contracted momentum, which is power suppressed in the collinear region. Therefore, the second terms in Eq. (6) can be dropped, when we focus on the infrared logarithms. The Feynman rules for the gauge bosons are then identical in the two phases in the collinear region with $k^{2} \rightarrow m_{W}^{2}$. Ghost propagators are also identical in the covariant gauge. Leptons remain massless in both phases at leading power. The above argument applies to the emissions of photons and $Z$ bosons. In the end, the Feynman rules for cross section calculations in both phases are made the same in the infrared region, implying that the infrared structure of the PDFs is not affected by the symmetry breaking at the scale $\mu_{s} \gg v$. That is, the matching of the PDFs at $\mu_{s}$ is perturbative, a key observation for the $\mathrm{EW}$ factorization in the high energy limit.

We elaborate on the connection between longitudinally polarized $W$ bosons in the broken phase and charged scalars in the unbroken phase. The longitudinal polarization vector of the former can be well approximated by $k^{\mu} / m_{W}$ at high energy below $\mu_{s}$. When this vector is contracted with the lepton-gauge-boson vertex, the Ward identity yields the suppression by powers of $m_{\ell} / v, m_{\ell}$ being a lepton mass. In the unbroken phase, scalar emissions from the lepton are suppressed by the tiny Yukawa coupling, equivalent to $m_{\ell} / v$ in the broken phase. This is a consequence of the Goldstone equivalence theorem [22]. Corrections to this theorem observed in [14] are actually of higher power, even though they are numerically important around the EW scale. Note that collinear gauge boson emissions from scalars are of leading power, while collinear scalar emissions from gauge bosons and fermions are power suppressed. The scalar PDFs remain of higher power under the evolution with the factorization scale, which involves either higher power initial conditions or splitting kernels.

The factorization formula for $e^{-} e^{+} \rightarrow \mu^{-} \mu^{+}+X$ takes the form

$$
\begin{aligned}
\frac{d \sigma^{\mu^{+} \mu^{-}}}{d p_{T} d y}= & \sum_{i, j=\ell, b} \int d x_{i} d x_{j} \phi_{i / e^{+}}\left(x_{i}, \mu\right) \phi_{j / e^{-}}\left(x_{j}, \mu\right) \\
& \times H_{i j \rightarrow \mu^{+} \mu^{-}+X}\left(x_{i}, x_{j}, \mu\right)+\mathcal{O}(v / E),
\end{aligned}
$$

where $b=W, \gamma, Z, \gamma Z$, and the hard function $H_{i j \rightarrow \mu^{+} \mu^{-}+X}$ describes the muon-pair production with the transverse momentum $p_{T}$ and the rapidity $y$ at the parton level. The double logarithms are absent in the EW evolution of $\phi_{\ell / e}$ as a consequence of the soft cancellation illustrated before, once the up- and down-type fermion distributions in $[15,16]$ are added. The standard PDFs from the EW evolution $[14,15,23]$ to a high scale $\mu>v$ can serve as the inputs of our PDFs in Eq. (7) according to the isospin sum. The PDFs at the parton level up to one loop can be obtained via the LO EW splitting functions, for example,

$$
\begin{aligned}
\phi_{\ell \mid \ell}(x, \mu)= & \delta(1-x)+\left(\frac{3}{4} g^{2}+g^{\prime 2} Y^{2}\right) \frac{1}{8 \pi^{2}} \ln \left(\frac{\mu^{2}}{v^{2}}\right) \\
& \times\left[\frac{1+x^{2}}{(1-x)_{+}}+\frac{3}{2} \delta(1-x)\right],
\end{aligned}
$$

from $\ell \rightarrow \ell+b$, in which $Y$ is the hypercharge, and the collinear logarithmic pieces are the same in both the broken and unbroken phases.

To conclude, we have explored the infrared structure of EW emissions in electron-positron collisions in the high energy limit, and proposed, for the first time, to handle EW shower effects from the viewpoint of factorization theorem. At ultra high energy, the significant EW shower makes an initial electron a beam of isospin doublets, that allows the cancelation of soft EW radiation in the isospin sum and the universal definitions of its $S U(2)$-singlet PDFs. We have pointed out that these PDFs possess the same collinear logarithms in the broken and unbroken phases, and that the perturbative matching at the EW symmetry breaking scale 
is a new feature compared to the QCD factorization. It was stressed that the up- and down-type fermion distributions considered separately in the literature do not respect the universality. For high-energy collisions involving protons, a quark PDF of the proton should be defined with the summation over colors and isospins. Besides, scalar emissions from the top quark parton can induce leading-power infrared logarithms because of its large Yukawa coupling, implying that the scalar PDFs of a proton need to be introduced. Our formalism provides a theoretical framework for collider physics far above the EW scale, and abundant extensions and applications are foreseen.

\section{ACKNOWLEDGMENTS}

We thank C. Bauer, J. M. Chen, T. Han, M. Mangano, E. Mereghetti, and V. Vaidya for helpful discussions. Y.-T. Chien thanks Los Alamos National Laboratory for the support during which part of this work was performed. This work was supported in part by the Ministry of Science and Technology of R.O.C. under Grant No. MOST-1042112-M-001-037-MY3, the U.S. Department of Energy (DOE), Office of Science under Contract No. DE-AC5206NA25396, the DOE Early Career Program and the LHC Theory Initiative Postdoctoral Fellowship under the National Science Foundation Grant No. PHY-1419008.
[1] N. Arkani-Hamed, T. Han, M. Mangano, and L.-T. Wang, Phys. Rep. 652, 1 (2016).

[2] J. Tang et al., arXiv:1507.03224.

[3] M. Mangano, CERN Yellow Report No. CERN 2017-003-M.

[4] A. Hook and A. Katz, J. High Energy Phys. 09 (2014) 175.

[5] P. Ciafaloni, D. Comelli, A. Riotto, F. Sala, A. Strumia, and A. Urbano, J. Cosmol. Astropart. Phys. 03 (2011) 019.

[6] M. Baumgart, I. Z. Rothstein, and V. Vaidya, Phys. Rev. Lett. 114, 211301 (2015).

[7] M. Bauer, T. Cohen, R. J. Hill, and M. P. Solon, J. High Energy Phys. 01 (2015) 099.

[8] G. Ovanesyan, T. R. Slatyer, and I. W. Stewart, Phys. Rev. Lett. 114, 211302 (2015).

[9] P. Ciafaloni and D. Comelli, Phys. Lett. B 446, 278 (1999).

[10] J.-y. Chiu, F. Golf, R. Kelley, and A. V. Manohar, Phys. Rev. Lett. 100, 021802 (2008).

[11] F. Bloch and A. Nordsieck, Phys. Rev. 52, 54 (1937).

[12] M. Ciafaloni, P. Ciafaloni, and D. Comelli, Phys. Rev. Lett. 84, 4810 (2000).
[13] G. Cacciapaglia, C. Csaki, and S. C. Park, J. High Energy Phys. 03 (2006) 099.

[14] J. Chen, T. Han, and B. Tweedie, J. High Energy Phys. 11 (2017) 093.

[15] C. W. Bauer, N. Ferland, and B. R. Webber, J. High Energy Phys. 08 (2017) 036.

[16] C. W. Bauer, N. Ferland, and B. R. Webber, arXiv: 1712.07147.

[17] L. A. Cavasonza, M. Kramer, and M. Pellen, J. Cosmol. Astropart. Phys. 02 (2015) 021.

[18] A. Denner and S. Pozzorini, Eur. Phys. J. C 18, 461 (2001).

[19] A. Denner and S. Pozzorini, Eur. Phys. J. C 21, 63 (2001).

[20] T. Kinoshita, J. Math. Phys. 3, 650 (1962).

[21] T. D. Lee and M. Nauenberg, Phys. Rev. 133, B1549 (1964).

[22] B. W. Lee, C. Quigg, and H. B. Thacker, Phys. Rev. D 16, 1519 (1977).

[23] P. Ciafaloni and D. Comelli, J. High Energy Phys. 11 (2005) 022. 